\title{
Pulling the trigger on the living kind module
}

Todd, Peter M. López, Alejandro

\section{Commentary on:}

S. Atran, Folk biology and the anthropology of science: Cognitive universals and cultural particulars. Behavioral and Brain Sciences. Vol 21,4 (1998), 547-609.

\author{
Please note: \\ This paper is a preprint of an article published in \\ Behavioral and Brain Sciences, 21 (4), 1998, \\ therefore there may be minor differences between \\ the two versions. \\ The copyright of this electronic version remains with \\ the authors and the Max Planck Institute for Human \\ Development.
}

\begin{abstract}
Atran speculates that a triggering algorithm for a living kind module could involve inputs from other modules that detect animacy and intentionality. Here we further speculate how algorithms for detecting specific intentions could be used to trigger between- or within-species categorization. We further indicate why such categorization may be adaptively important in Eldredge's energy and information realms.
\end{abstract}

How might we expect the human mind to be designed to carve up the natural world? On the one hand, those species that Atran indicates humans most readily perceive - vertebrates and flowering plants - have evolved to be distinguishable largely through the categorizing abilities of brains and perceptual systems (Todd \& Miller, 1997). Animal species are generally self-defining entities, kept reproductively isolated from other species through sexually selected traits and the nervous systems that evaluate and make choices based on them. Many salient aspects of flowering plants have similarly been shaped through coevolution with the categorization mechanisms of the animal species that pollinate them. Thus it is perhaps not surprising that our own psychology has evolved to apprehend and appreciate the diversity of species created by the selective action of other behaving organisms.

On the other hand, there is no reason to reason - to make connections between categories, or even to categorize the world at all - if such mental work does not serve an adaptive function. That is, we should not expect to be designed to think about the natural world in terms of categories of other species, and individuals within our own species, unless these distinctions helped us with the adaptive tasks of survival and reproduction. Atran indicates that knowing the habits and relationships of different species "would be likely to increase the effectiveness (benefit) of such knowledge-based subsistence immeasurably" - but can we be any more specific than this? If we can say more clearly when species-level categorization might give an individual an adaptive benefit, we could also form new hypotheses about when and how this type of domain-specific reasoning could be triggered.

Eldredge (1986) distinguishes two domains in which all organisms must function: the energy domain, procuring the physical elements necessary for survival, and the information domain, ensuring the spread of copies of one's own genetic makeup via reproduction. Other species are (usually) only of concern in the former domain: they can be a source of body-building compounds as prey, or of energy-draining insult as predators, but not of additional genetic information for recombination and reproduction. Instead, only members of one's own species can provide such relevant genetic information, so they are crucial for the second domain. Energy and physical resources are not individuated - they are essentially the same in all members of a given species - so there is no need to distinguish between members of another species. In contrast, genetic information is unique to individuals, and so it matters who is chosen as a mate, or with whom one engages in preferential treatment (i.e., offspring and kin). This difference of individuation between the energy and information realms can explain the adaptiveness of categorizing at the species level outside one's own kind and at the individual level within.

Thus, species-level categorization will primarily be invoked, not surprisingly, in adaptive problems contained within the energy/resource/survival domain. Within that domain, it is important to know when such an adaptive problem is being faced - for instance, when a predator is threatening, or when a prey animal is fleeing. These are the instances in which it may be most important to generalize behavioral knowledge about the species as a whole to the current situation at hand. We have explored a simple visual-cue-based algorithm for judging intention from motion in just such instances (Blythe, Miller, \& Todd, 1996). We had participants generate motions of two moving "bugs" on a computer screen, corresponding to simple intentional categories including pursuit, evasion, fighting, courtship, and play. Other participants were later able to categorize the intentions of the "bugs" with high accuracy from their trajectories alone. This study supports the notion that animate intention can be determined using only a few simple spatiotemporal cues (which include, from trajectory analysis, relative heading, relative distance, relative velocity, and vorticity or "loopiness"). Knowing the intention (as opposed to the general intentionality that Atran mentions) of another organism can trigger the appropriate domainspecific mechanism for response, including species-level categorization and recall of relevant traits.

It is of course also important to know the intentions of other organisms within one's own species, which can have an impact in the information or energy domains (e.g., courting or fighting, respectively). In humans, these intentions can be revealed - and the appropriate domain-specific reactions triggered - not only through gross bodily movements, but also through verbal cues. We have developed a simple, "fast and frugal" intention-judging algorithm based on this type of linguistic information as well (Lopez, Davis, \& Todd, in preparation). While this algorithm does not directly connect with Atran's core module for carving up the natural world of other species, we mention it here to provide another example of how a simple triggering algorithm can invoke further domain-specific reasoning mechanisms, and to point out the importance of this kind of higher-level "director" mechanism in human cognition. 
The Pragmatic Cues (PC) algorithm maps conditional statements - if $\mathrm{P}$, then $\mathrm{Q}$ - onto social domains. Humans regularly use such conditional statements to make promises and threats, offer advice and warnings, give permissions and obligations to other people. But how do we know when we are facing a promise, or a threat, or something else? According to the PC algorithm, we simply traverse a binary decision tree based on just three pragmatic cues that sequentially prune the possibilities until a single social domain is left. The cues are the following: Is $Q$ (the consequent) a benefit for the speaker? Does Q involve an act of the speaker? Does Q enable or obligate an act of the listener? Given just these three simple cues, the relevant social domain can be determined - the PC algorithm categorized the conditionals in our test-set onto social domains five times better than chance and just slightly worse than people (average across domains was $85 \%$ for the algorithm, and $94 \%$ for human raters).

Mental mechanisms like these for triggering domain-specific behaviors, whether based on motion cues of other species or verbal cues from our own species, must be fast and, hence, use as little information as they can get away with (Gigerenzer \& Todd, in press). As a consequence, as Atran indicates, these simple algorithms can sometimes make mistakes, leading us, for example, to overgeneralize animacy and intention to moving computer images. But such false alarms are probably a lower-cost mistake than missing the cues of animacy of real organisms in the environment. In our rather environmentally impoverished intention-from-motion studies, some participants were certain that the "bug" they were seeing on a computer screen was being moved by a program on the computer itself, rather than by another participant in a separate room (as was the case); but in the jungle, to mistake the movement of leaves as caused by the wind rather than the jaguar lurking there could be costly indeed.

\section{References}

Blythe, P.W., Miller, G.F., and Todd, P.M. (1996). Human simulation of adaptive behavior: Interactive studies of pursuit, evasion, courtship, fighting, and play. In P. Maes, M.J. Mataric, J.-A. Meyer, J. Pollack, and S.W. Wilson (Eds.), From animals to animats 4: Proceedings of the Fourth International Conference on Simulation of Adaptive Behavior (pp. 13-22). Cambridge, MA: MIT Press/Bradford Books.

Eldredge, N. (1986). Information, economics, and evolution. Annual Review of Ecology and Systematics, 17, $351-369$.

Gigerenzer, G., and Todd, P.M. and the ABC Research Group (Eds.) (1999). Simple heuristics that make us smart. New York: Oxford University Press.

Lopez, A., Davis, J.N., and Todd, P.M. (in preparation). "If...": Satisficing algorithms for mapping conditional statements onto social domains.

Todd, P.M., and Miller, G.F. (1997). Biodiversity through sexual selection. In C.G. Langton and K. Shimohara (Eds.), Artificial Life V: Proceedings of the Fifth International Workshop on the Synthesis and Simulation of Living Systems (pp. 289-299). Cambridge, MA: MIT Press/Bradford Books. 